\title{
Negative Refraction Using Frequency-Tuned Oxide Multilayer Structure
}

\author{
Yalin Lu, ${ }^{1}$ Gail J. Brown, ${ }^{2}$ and Kitt Reinhardt ${ }^{3}$ \\ ${ }^{1}$ Laser and Optics Research Center (LORC), Department of Physics, U.S. Air Force Academy, CO 80840, USA \\ ${ }^{2}$ AFRL/ RXPSO, Wright-Patterson Air Force Base, Building 651, 3005 Hobson Way, OH 45433, USA \\ ${ }^{3}$ AFOSR/NE, Suite 326, 875 North Randolph Street, Arlington, VA 22203, USA
}

Correspondence should be addressed to Yalin Lu, yalin.lu@usafa.edu

Received 14 May 2008; Accepted 31 August 2008

Recommended by Hiroshi Murata

\begin{abstract}
An oxide-based multilayer structure was proposed to realize negative refraction. The multilayer composes of alternative layers having negative permittivity and negative permeability, respectively. In order to realize negative refraction, their dielectric and magnetic resonances of layers will be tuned to the frequency as close as possibly via changing their temperature, composition, structure, and so forth. Such oxide-based NIMs are attractive for their potential applications as optical super lenses, imagers, optical cloaking, sensors, and so forth, those are required with low-loss, low-cost, and good fabrication flexibility.
\end{abstract}

Copyright () 2008 Yalin Lu et al. This is an open access article distributed under the Creative Commons Attribution License, which permits unrestricted use, distribution, and reproduction in any medium, provided the original work is properly cited.

\section{Introduction}

Negative index materials (NIMs) have attracted extensive attention in recent years since the first observation of negative refraction in those artificially made metamaterials consisting of arrays of metallic rings/rods [1]. Majority of such NIMs use metallic split ring resonance (SRR), surface plasmonic resonance, or a combination of magnetic and metallic materials with naturally occurring negative permeability $(\mu)$ or negative permittivity $(\varepsilon)$. Very unique properties of such metamaterials, including negative refraction, have been previously demonstrated mainly for operation in microwave frequencies. A new trend of current NIM research is toward the optical frequency. For example, a lossless negative index slab material could act as a "superlens" or a "perfect lens," which could image object with a high resolution far below the conventional optical diffraction limit, and are attractive to those applications including data storage, medical imaging, biophysics, and so forth. In addition to those metamaterials, negative refraction has also been found in other material formats including planar transmission lines, photonic crystals, and so forth. However, all such NIMs use complicated artificial structures requiring high-precision nanofabrication and can hardly be rigorously considered as uniform materials, since the size of their structural elements is usually greater than one tenth of the operation wavelength. In addition, certain polarization and incident angle of an electromagnetic (EM) field must be maintained in order to realize such negative refraction.

Considering the fact that an SRR in a metamaterial produces $-\varepsilon \psi$ and $-\mu \psi$, respectively [2], such a metamaterial will be similar to a multilayer composed by alternating $-\varepsilon \psi$ and $-\mu \psi$ layers-the later will be much easier to realize as a natural material. It is also well known that some natural materials have inherent $-\varepsilon \psi$ or $-\mu$, which will be useful for multilayer NIM fabrications. For example, certain ferrites have $-\mu$ in microwave frequencies, and many conducting materials have $-\varepsilon$ below the plasma frequency. Studies toward this direction have been performed, mostly by simulations, in stacked ferrite-dielectric waveguides, ferrite-metal, ferromagnetic-superconductor, and ferritesemiconductor multilayers [3]. However, the use of conductive materials may excite surface waves at their interfaces, which in turn change their electromagnetic characters, and more seriously, result in a significant high-loss concern. Apparently, an NIM composing of nonconductive optical materials will be promising.

Availability of the frequency tunability to an optical NIM will provide a much bigger freedom in exploring its potential applications as sensors, modulators, switches, and 
so forth. Variable electromagnetic response when changing a metamaterial's structure is not a practical way of frequency tuning. External tuning via filling liquid crystals and photoconductive materials to metamaterials will work for some frequencies. It is normally slow and has concern on both reliability and stability of performances.

This manuscript intends to discuss one possible mechanism potential able to realize negative refraction using all oxide-base materials in the format of multilayer. Such oxidebased negative index multilayers are expected to potentially have low optical losses, against to those existing designs using conductive materials, and are potentially frequency-tunable if the NIM's composing materials are dielectrically tunable by external fields including electric, optical, and microwave. Tunable dielectrics are easily realizable in many piezoelectric and piezomagnetic complex oxides. Therefore, the impact of such NIMs to their potential applications such as super lenses, optical cloaking, optoelectronics, and sensing can be significant.

\section{Oxide Multilayer NIMs}

The mechanism to build the oxide multilayer NIM to be discussed is a resonance-tuned multilayer (RTM) composing of alternative dielectric and magnetic oxide layers having naturally occurring $-\varepsilon \psi$ or $-\mu$, respectively, and having their resonance frequencies finely tuned to concurrence via both structural and compositional engineering to both used materials.

The RTM mechanism considers the nonlattice-matched multilayer case and the two alternative oxide layers as the multilayer have $-\mu$ or $-\varepsilon$, respectively. For example, the oxide multilayer can be represented as $(A / B)_{m}$, and " $A$ " has an intrinsic dielectric resonance, and " $B$ " represents a material with an intrinsic magnetic resonance. The RTM approach will be used to fine-tune the two resonance frequencies concurrence together, and realize the negative refraction immediately after the merged resonance frequency. Fine-tuning to each oxide material can be realized by changing composition, structure, temperature, magnetic field, and so forth. Schematic of the RTM NIM is shown in Figure 1. In Figure 1, only two adjacent laminas (one dielectric and the other magnetic) having thickness of $d_{1}$ and $d_{2}$, respectively, are shown. They will be the building block for further making into the multilayer structure. The insets on the right side schematically show the frequency spectra of both permittivity and permeability around the resonance frequencies. Arrows indicate the directions of frequency tuning to make two resonance frequencies concurrence. Figure 2 shows the schematic multilayer structure and the concurrence-occurred frequency spectra showing the frequency region with both negative permittivity and negative permeability.

In order to achieve a concurrence of both dielectric and magnetic resonances in dielectric and magnetic oxide materials, different origins of dielectric and magnetic responses have to be considered for different frequencies ranging from the UV/visible, far-infrared (including the major terahertz band), to microwave. For the UV to visible spectrum,

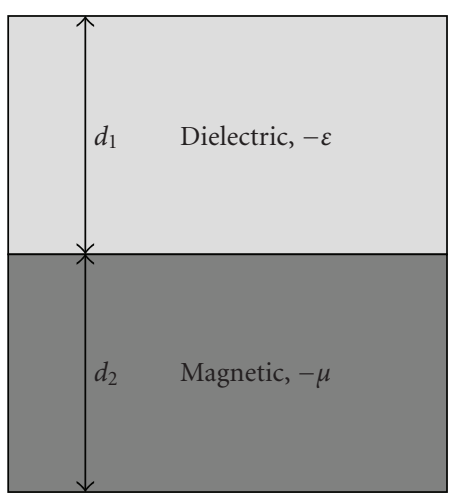

Building block for the multilayer

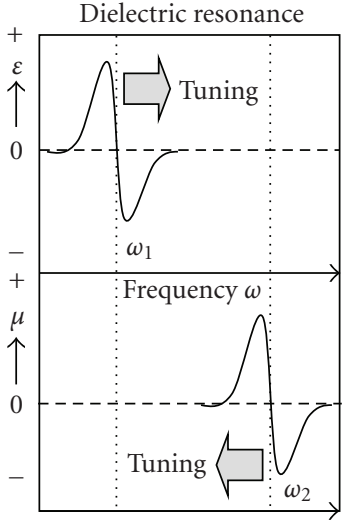

Magnetic resonance
FIgURE 1: Schematic of a negative permittivity and negative permeability double layer (the building block for multilayer, the left side of the figure) and tuning of their resonance frequencies to concurrence-occur to the same frequency (inset at the right side).

for example, the dielectric response is primarily from the electronic polarization modes and the magnetic response from the magnetic dipoles. In the far-IR range, ionic polarization modes, and antiferromagnetic resonances (AFMRs) are mainly responsible for dielectric and magnetic responses, respectively. Similarly, for those microwave frequencies, electronic dipole reorientation (domain wall moving) and ferromagnetic resonances (FMRs) are the main causes.

Other than the resonance frequency of the concerning materials, both degree of damping and amplitude of dielectric and magnetic responses will also play the key rule. For example, in optical frequencies, direct magnetic dipole response is orders of magnitude weaker than electronic dipole response. The above-discussed SCS mechanism actually provides an indirect coupling of the magnetic dipole response to a strong electronic dipole resonance via the phonon resonance, yielding both negative permeability and negative permittivity after the phonon resonance frequency.

The dielectric dispersion due to the electronic dipole polarization can be fitted to the following empirical expression:

$$
\frac{\varepsilon^{*}(\omega)-\varepsilon_{\infty 1}}{\varepsilon_{0}-\varepsilon_{\infty 1}}=\sum_{i=1}^{n} g_{i}\left[\frac{1}{\left(1+i \omega \tau_{i}\right)}\right],
$$

where $\varepsilon_{\infty 1}$ is the permittivity due to the ionic polarization, $\varepsilon_{0}$ is the low-frequency permittivity, $\omega$ is the angular frequency, and $g_{i}$ is the distribution of relaxation time $\left(\tau_{i}\right)$. On the other hand, the dielectric dispersion due to the ionic polarization can be fitted to the following four-parameter semiquantum model [4]:

$$
\varepsilon^{*}(\omega)=\varepsilon_{\infty 2} \prod_{j=1}^{n} \frac{\omega_{j \mathrm{LO}}^{2}-\omega^{2}+i \gamma_{j \mathrm{LO}} \omega}{\omega_{j \mathrm{TO}}^{2}-\omega^{2}+i \gamma_{j \mathrm{TO}} \omega}
$$

where $\varepsilon_{\infty 2}$ is the permittivity due to the electronic polarization, $n$ is the number of optical phonon mode, $\gamma$ is the damping factor, and TO and LO represent transverse 


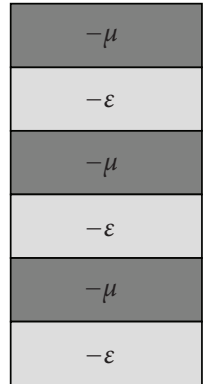

Frequency-tuned multilayer

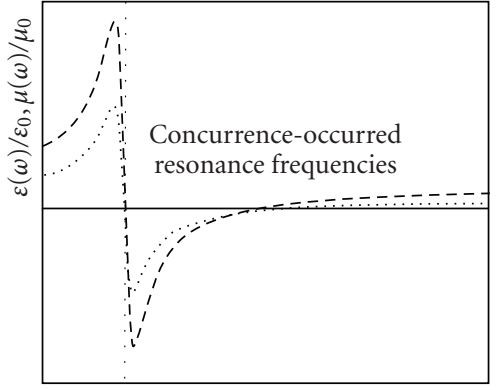

Frequency

$$
\begin{gathered}
\ldots . . \\
\ldots(\omega) \\
---\mu(\omega)
\end{gathered}
$$

Figure 2: Schematic of the frequency-tuned multilayer structure (left) and the concurrence-occurred resonances on both permittivity and permeability (right). After the concurrence-occurred resonance frequency, both negative permittivity and negative permeability will occur, which indicates negative optical refraction.

and longitudinal optical phonon modes, respectively. The relationship between the $\varepsilon^{*}$ and IR reflectivity $R(\omega)$ can be given by

$$
R(\omega)=\left|\frac{\left(\sqrt{\varepsilon^{*}(\omega)}-1\right)}{\left(\sqrt{\varepsilon^{*}(\omega)}+1\right)}\right| .
$$

Equations (1), (2), and (3) permit a direct identification of the dielectric spectra and resonance frequencies via the experimental IR reflectivity measurement on a specific dielectric material system. Contribution from domain wall relaxation is usually masked by electronic and ionic relaxations, unless the temperature is close to the material's Curie temperature or phase transition temperature. It has been usually reported at above $100 \mathrm{kHz}$ frequencies, will be minor to those optical frequencies of our current discussion interest.

Magnetic resonances can be identified from recording the complex permeability spectra on those magnetic oxide materials of interests. Accordingly, the complex permeability can be expressed as a superposition of two contributions, $\mu(\omega)=1+\tilde{\chi}_{\mathrm{dw}}(\omega)+\tilde{\chi}_{\mathrm{rot}}(\omega)$, namely, the domain wall component $\tilde{\chi}_{\mathrm{dw}}(\omega)$ and the magnetic moment rotation $\tilde{\chi}_{\text {rot }}(\omega)$ :

$$
\begin{aligned}
& \tilde{\chi}_{\mathrm{dw}}(\omega)=\frac{\chi_{\mathrm{dw}} \omega_{\mathrm{dw}}^{2}}{\omega_{\mathrm{dw}}^{2}-\omega^{2}+j\left(\beta / m_{\mathrm{dw}}\right) \omega}, \\
& \tilde{\chi}_{\mathrm{rot}}=\frac{\left(\omega_{\mathrm{rot}}+j \alpha \omega\right) \chi_{\mathrm{rot}} \omega_{\mathrm{rot}}}{\left(\omega_{\mathrm{rot}}+j \alpha \omega\right)^{2}-\omega^{2}},
\end{aligned}
$$

where $\chi_{\mathrm{dw}}$ and $\chi_{\text {rot }}$ are static (or low frequency) susceptibilities for domain wall (DW) and magnetic moment rotation (ROT) motions, $\omega_{\mathrm{dw}}$ and $\omega_{\text {rot }}$ are resonance frequencies of DW and ROT components, $\beta$ and $\alpha$ are corresponding damping factors, $m_{\mathrm{dw}}$ is the effective mass of wall, and $\omega$ is the frequency of the driving ac magnetic field. Description of magnetic moment motions will be highly dependent on materials. For simplicity, we consider both AFMR and FMR cases which will be similar in theory.
In a magnetic material having a uniaxial anisotropy and the magnetic field is applied parallel to the easy axis, the AFMR frequency $\omega_{\mathrm{AFMR}}$ at low temperature (below Néel temperature) is given by

$$
\begin{array}{ll}
\frac{2 \pi \omega_{\mathrm{AFMR}}}{\gamma}=\sqrt{2 H_{E} H_{A}} \pm H, & \left(H<H_{\mathrm{SF}}\right), \\
\frac{2 \pi \omega_{\mathrm{AFMR}}}{\gamma}=\sqrt{H^{2}-2 H_{E} H_{A}}, \quad\left(H>H_{\mathrm{SF}}\right),
\end{array}
$$

where $\gamma\left(\equiv g \mu_{B} / \hbar\right)$ is the magnetomechanical ratio, $H_{E}$ is the exchange field, $H_{A}$ is the anisotropy field, and $H_{\mathrm{SF}}$ is the spinflop field. When $H$ is applied perpendicular to the easy axis, then the $\omega_{\mathrm{AFMR}}$ is given by

$$
\frac{2 \pi \omega_{\mathrm{AFMR}}}{\gamma}=\sqrt{H^{2}+2 H_{E} H_{A}} .
$$

For FMR, a simple expression about the $\omega_{\mathrm{FMR}}$ is Kittel's equation

$$
\frac{\omega_{\mathrm{FMR}}}{\gamma}=\sqrt{\left(H_{A, \mathrm{eff}}+H_{E}\right) 4 \pi M_{s}}
$$

where $H_{A, \text { eff }}$ is the effective anisotropy field, and $4 \pi M_{s}$ the saturation magnetization. For both AFMR and FMR cases and when for qualitative analyses, it can be simplified to $\omega \propto$ $\sqrt{H_{A} H_{E}}$.

Depending on the material selection for such multilayer, the above equations provide the way to measure their resonance frequencies via a variety of approaches including optical reflection, time-domain terahertz $(\mathrm{THz})$ transmission, magnetic analysis, and so forth. The above equations also theoretically provide the direction to realize the efficient frequency tuning in order to achieve concurrence of both dielectric and magnetic resonance frequencies.

\section{Frequency Tuning}

Tuning both dielectric and magnetic resonance frequencies may follow a few different ways. In certain ferroelectric materials, optical mode of the lattice (phonon) is dependent on both temperature $(T)$ and the material's Curie temperature $\left(T_{c}\right)$, roughly following an empirical relation: $\omega^{2} \propto\left(T-T_{c}\right)$ as an example. As an example, Figure 3 shows the dependence of relative dielectric constant on the composition. The dielectric maximum occurs at $x_{\mathrm{Ba}} \sim 0.7$ [at where the ferroelectric-paraelectric boundary (FPB) occurs], and it Curie temperature changes from $415 \mathrm{~K}\left(x_{\mathrm{Ba}} \sim 1\right)$ to about $40 \mathrm{~K}\left(x_{\mathrm{Ba}} \sim 0\right)$ almost linearly [5]. Similarly, tuning by changing both temperature and material composition is also applicable to some magnetic oxide materials. Inset in Figure 2 show a general frequency tuning schematic via changing the temperature, in order to reach a concurrence at the same frequency for both dielectric and magnetic resonances. Apparently, tuning their compositions to reach a room temperature operation of the NIMs will be the most attractive, simply because of the practicability for applications. 


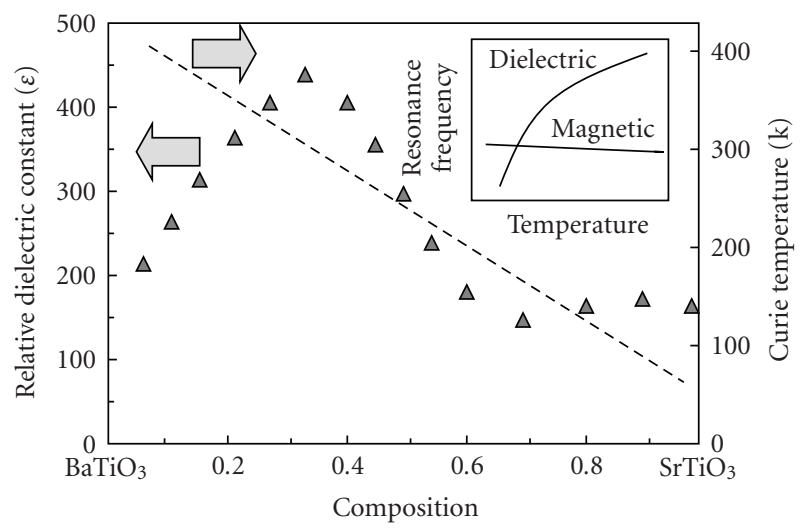

Figure 3: Dependence of the dielectric constant and Curie temperature of $(\mathrm{Ba}, \mathrm{Sr}) \mathrm{TiO}_{3}$ on its composition. Inset shows a possible frequency tuning by changing the temperature for reaching the concurrence of both dielectric and magnetic resonance frequencies.

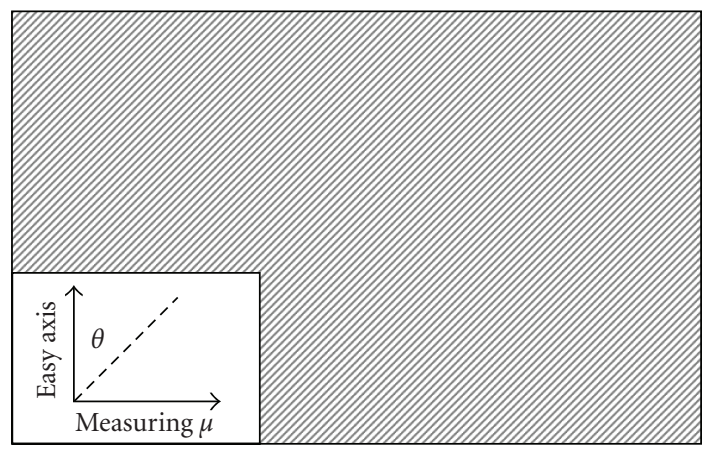

FIGURE 4: Schematic of the multilayer structure alignment required for possibly tuning the FMR resonance frequency via changing the structural parameters-width, period, duty cycle, and the orientation angle.

An alternative way of possibly tuning the magnetic resonance frequency is by changing the magnetic layer's alignment inside the multilayer structure. Figure 4 shows the schematic of such a structure in which the FMR frequency will be tuned by changing either the orientation angle $\theta$ or the layer thickness, following the rewritten Kittel's formula involving both uniaxial anisotropy field $\left(H_{u}\right)$ and the additional shape anisotropy field $\left(H_{k, \text { eff }}\right)$ into the total effective anisotropy field $\left(H_{A, \text { eff }}\right)$

$$
\begin{aligned}
& H_{A, \text { eff }}=H_{u}+H_{k, \text { eff }} \cos \theta, \\
& \frac{2 \pi \omega_{\text {AFMR }}}{\gamma}=\sqrt{4 \pi M_{s} H_{A, \text { eff. }}}
\end{aligned}
$$

In Figure 4, the main picture shows the parallel strait structure to be made in the magnetic layer inside the superlattice, via selective etching the as-fabricated magnetic layer or mask-shielding growth, and so forth. The inset shows that when one measures the permeability horizontally, the result will be actually tunable by changing the strait's size (both period and width) or by changing the angle respective to the easy axis of the magnetic material. The multilayer structure itself to be discussed in this theory actually provides the great easiness to reach such frequency tuning for the magnetic part inside the multilayer. Structural parameters that can be varied when designing include the layer thickness, periodic duty cycle, and magnetic orientation, and so forth. An efficient way to optimize material compositions and structural parameters may follow the combinatorial strategy [6]. Effort toward efficient material selection, simulation for frequency tuning, and the multilayer fabrication is ongoing and will be published separately.

\section{Conclusion}

One possible physical mechanism toward realizing negative refraction in a fully oxide-based multilayer was theoretically discussed. Inside the multilayer structure, such negative refraction may be achieved by tuning their resonances to the same frequency via changing temperature, layer composition, structural parameter, and so forth. Such oxidebased NIMs are attractive for their potential applications as optical super lenses, imagers, sensors, and so forth, that are potential with low-loss, cost-effectiveness, and good fabrication flexibility.

\section{References}

[1] R. A. Shelby, D. R. Smith, and S. Schultz, "Experimental verification of a negative index of refraction," Science, vol. 292, no. 5514, pp. 77-79, 2001.

[2] D. R. Smith, S. Schultz, P. Markoš, and C. M. Soukoulis, "Determination of effective permittivity and permeability of metamaterials from reflection and transmission coefficients," Physical Review B, vol. 65, no. 19, Article ID 195104, 5 pages, 2002.

[3] R. H. Tarkanyan and D. G. Niarchos, "Effective negative refractive index in ferromagnet-semiconductor superlattices," Optics Express, vol. 14, no. 12, pp. 5433-5444, 2006.

[4] F. Gervais, "High-temperature infrared reflectivity spectroscopy by scanning interferometry," in Infrared and Millimeter Waves, K. J. Button, Ed., vol. 8, pp. 279-339, Academic Press, New York, NY, USA, 1983.

[5] J. Li, F. Duewer, C. Gao, H. Chang, X.-D. Xiang, and Y. Lu, "Electro-optic measurements of the ferroelectric-paraelectric boundary in $\mathrm{Ba}_{1-x} \mathrm{Sr}_{x} \mathrm{TiO}_{3}$ materials chips," Applied Physics Letters, vol. 76, no. 6, pp. 769-771, 2000.

[6] Y. Lu, "The structural engineering strategy for photonic material research and device development," Active and Passive Electronic Components, vol. 2007, Article ID 17692, 7 pages, 2007. 

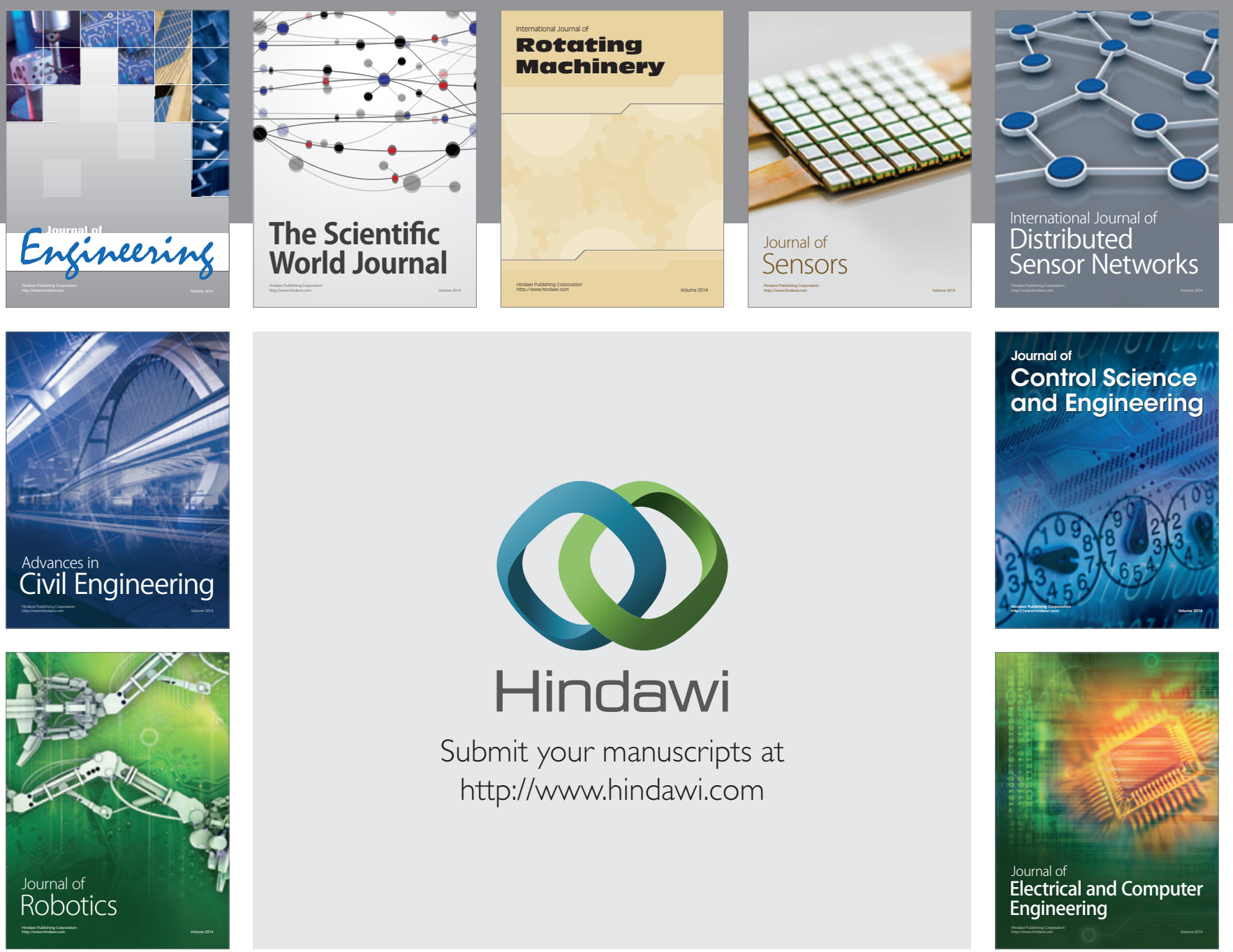

Submit your manuscripts at

http://www.hindawi.com
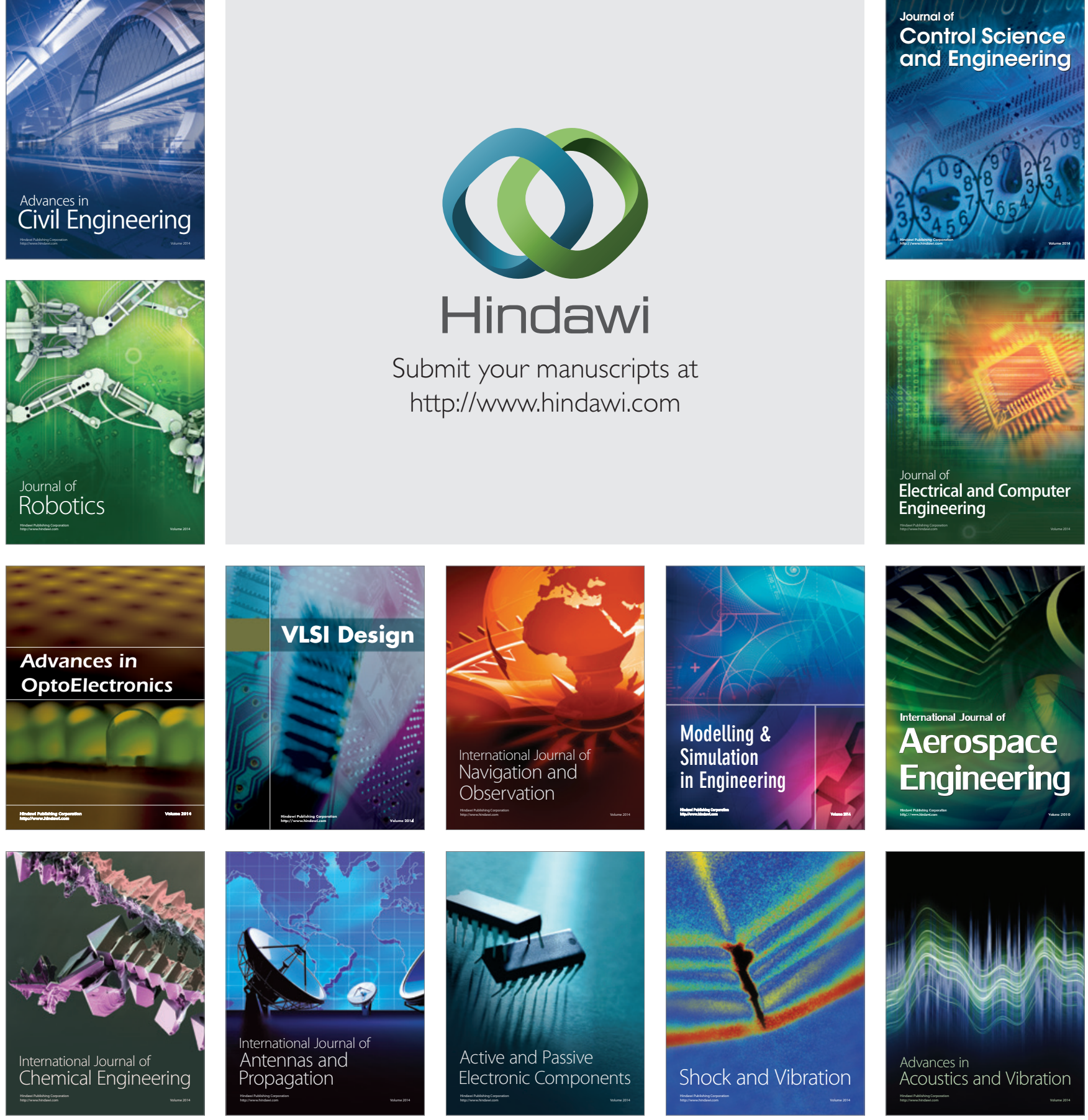